\title{
A novel algorithm to model the influence of host lattice flexibility in molecular dynamics simulations: Loading dependence of self-diffusion in carbon nanotubes
}

\author{
S. Jakobtorweihen ${ }^{\text {a) }}$ \\ Chemical Reaction Engineering, Hamburg University of Technology, Eissendorfer Strasse 38, \\ D-21073 Hamburg, Germany \\ C. P. Lowe \\ Van 't Hoff Institute for Molecular Sciences (HIMS), University of Amsterdam, Nieuwe Achtergracht 166, \\ 1018 WV Amsterdam, The Netherlands \\ F. J. Keil \\ Chemical Reaction Engineering, Hamburg University of Technology, Eissendorfer Strasse 38, \\ D-21073 Hamburg, Germany \\ B. Smit \\ Van 't Hoff Institute for Molecular Sciences (HIMS), University of Amsterdam, Nieuwe Achtergracht 166, \\ 1018 WV Amsterdam, The Netherlands and Centre Européen de Calcul Atomique Moléculaire \\ (CECAM), Ecole Normale Supérieure, 46 Allée d'Italie, 69364 Lyon Cedex 7, France
}

(Received 19 December 2005; accepted 14 February 2006; published online 18 April 2006)

\begin{abstract}
We describe a novel algorithm that includes the effect of host lattice flexibility into molecular dynamics simulations that use rigid lattices. It uses a Lowe-Andersen thermostat for interface-fluid collisions to take the most important aspects of flexibility into account. The same diffusivities and other properties of the flexible framework system are reproduced at a small fraction of the computational cost of an explicit simulation. We study the influence of flexibility on the self-diffusion of simple gases inside single walled carbon nanotubes. Results are shown for different guest molecules (methane, helium, and sulfur hexafluoride), temperatures, and types of carbon nanotubes. We show, surprisingly, that at low loadings flexibility is always relevant. Notably, it has a crucial influence on the diffusive dynamics of the guest molecules. (C) 2006 American Institute of Physics. [DOI: 10.1063/1.2185619]
\end{abstract}

\section{INTRODUCTION}

Carbon nanotubes (CNTs) are nanoscale materials with many promising properties (thermal, mechanical, and electronic). ${ }^{1,2}$ Since their discovery in $1991,{ }^{3}$ several novel and advanced synthesis methods have been suggested. ${ }^{1,4-6}$ Naturally, this has motivated studies of their application in many fields. One field among these is chemical engineering science, more specifically separation technology and catalysis. Carbon nanotubes (CNTs) can be aligned in a polymer film to form a well-ordered nanoporous membrane structure $^{7,8}$ that can be incorporated in a macroscopic structure $^{9}$ for separation devices. It is therefore of practical interest to understand the diffusive behavior of molecules adsorbed in these materials. ${ }^{10-14}$

The adsorption capacity and the fast diffusion of guest molecules inside CNTs were the motivations for studies of diffusion $^{10-12,14-37}$ and adsorption in CNTs (Refs. 18, 23, and 38-45) by means of molecular simulations. A particularly interesting observation is the remarkable increase in the diffusion coefficient of simple molecules at low densities observed by Skoulidas et al. ${ }^{10}$ These molecular simulations pre-

\footnotetext{
${ }^{a)}$ Author to whom correspondence should be addressed. Electronic mail: jakobtorweihen@tuhh.de
}

dicted a diffusion coefficient higher than the corresponding gas phase value. This pronounced increase was subsequently reproduced by other groups ${ }^{17,18}$ and explained in terms of the smoothness of the nanotube. ${ }^{10}$ In a recent Letter we investigated the assumptions underlying these simulations. ${ }^{15}$ In particular we focused on the differences between simulating diffusion in a nanotube in which the carbon atoms are effectively frozen (rigid lattice) and a model in which the carbon atoms undergo thermal motions. We showed that this CNT "flexibility" has a crucial influence on self-diffusion at low loadings. Simulating the CNT as a flexible framework resulted in diffusion coefficients an order of magnitude smaller. Furthermore, in the low loading region a plateau region that was not detected when simulating a rigid CNT was observed. In their recent publications Sholl and co-workers ${ }^{20-23}$ investigated the behavior of guest molecules over a wide range of loadings (down to the zero loading limit). They justified the rigid CNT assumption based on the work of Sokhan et al. ${ }^{16}$ Indeed Sokhan et al. showed that nanotube flexibility plays only a minor role. But they investigated the behavior inside CNTs for low Knudsen numbers $(0.02-0.17)$. This agrees with our observations. ${ }^{15}$ We could not observe a flexibility influence under these conditions. The influence of flexibility manifests itself in the low loading (high Knudsen numbers) regime. In Ref. 14 it was predicted 
that the collective diffusivity is also influenced by carbon nanotube flexibility.

Supple and Quirke ${ }^{28}$ studied the imbibition of a CNT with decane. They noted that pore flexibility had no influence on their results. Their study involved the filling of a CNT in contact with a liquid where transport diffusion and entrance effects are relevant. It is therefore not comparable to our work. Sinnott and co-workers ${ }^{29-32}$ simulated the carbon nanotube as a flexible framework. They studied entrance effects, transport diffusion, and molecular diffusion for various carbon nanotubes and various guest molecules. However, they did not explicitly study the role of flexibility. Bhide and Yashonath investigated the dynamics of different molecules in CNTs. ${ }^{33-36}$ In all cases they observed superdiffusive motion. This can be attributed to the way in which they analyzed the mean-squared displacement (see Sec. VI). In their studies the CNT was treated as being rigid. This is a widely made assumption. ${ }^{10,17-27}$

In none of the above mentioned works was the influence of CNT flexibility investigated systematically. Only for a few specific systems was it checked if flexibility had an influence. Zeolites are widely studied nanoporous materials. Interestingly, whether or not it is reasonable to assume a rigid lattice in adsorption ${ }^{46}$ or diffusion studies ${ }^{47}$ of zeolite systems is far from being understood. In a previous Letter we reported a flexibility influence on diffusion at low loadings in CNTs. ${ }^{15}$ The reason for the common approximation of a rigid lattice is the computationally demanding nature of simulations that treat the host matrix explicitly. A large number of intramolecular interactions must be calculated in one time step of a molecular dynamics simulation (large number of substrate atoms with high connectivity). To avoid these expensive simulations we introduced a novel and very efficient algorithm that takes the most important aspects of flexibility into account at a small fraction of the costs of a fully flexible CNT simulation. Our method results in effectively the same diffusivities and other effects obtained from the flexible CNT simulations. ${ }^{15}$ This algorithm can be applied to other confined systems (zeolites, ion channels, membranes, etc.). The central idea of this "Lowe-Andersen interface-fluid collision (LA-IFC) thermostat" is to mimic the thermal effects of a flexible framework stochastically. Very recently, Chen et al. ${ }^{48}$ applied this thermostat to calculate transport diffusion in CNTs. They observed a distinct influence of flexibility on transport diffusion. In another recent publication the influence of flexibility on the hydrophobic behavior of CNTs was revealed. ${ }^{49}$ Furthermore, it was shown that flexibility decreases the mobility of water. This agrees with our observations.

The aim of this work is to systematically study the influence of CNT flexibility on self-diffusion. Furthermore, we describe the new algorithm for incorporating the flexibility effect into a simulation of a rigid CNT in detail. In addition, we examine the flexibility influence for different CNTs and different guest molecules $\left(\mathrm{CH}_{4}, \mathrm{He}\right.$, and $\left.\mathrm{SF}_{6}\right)$. The outline of this paper is as follows. In the next section the models underlying our simulations are explained. Details of the simulations are given in Sec. III. In Sec. IV the effect of flexibility on diffusion is discussed and our new algorithm is explained in detail, while in Sec. V results obtained with the thermal diffuse scattering ${ }^{50}$ algorithm are compared to results obtained with the LA-IFC thermostat. In Sec. VI results of self-diffusion for different components in different carbon nanotubes are shown. Mainly the pore size and temperature influence is investigated. We summarize our results in the last section.

\section{THE MODEL}

In this work we are interested in the dynamics of simple gases in carbon nanotubes. Here we describe the various models.

\section{A. Carbon nanotubes}

Carbon nanotubes are nanometer-scale pores that can be viewed as rolled up graphite. The size and helicity of carbon nanotubes are specified by two integer numbers $n_{1}$ and $n_{2},{ }^{1,2}$ so they can be unambiguously labeled as $\left(n_{1}, n_{2}\right)$. For $n_{1}$ $=n_{2}$ the CNT is in an armchair structure (one carbon-carbon bond perpendicular to the pore axis), and for $n_{2}=0$ it is in a zigzag structure (one carbon-carbon bond parallel to the pore axis). All results in this work were obtained with single walled carbon nanotubes in a zigzag structure. Differences in helicity do not affect the dynamics of adsorbents in carbon nanotubes. $^{10,30}$

\section{B. Flexible carbon nanotubes}

To model a carbon nanotube as a flexible framework we followed Walther et al. ${ }^{51}$ Bond vibrations were modeled with a Morse potential:

$$
U_{\text {vib }}=K_{\text {vib }}\left(e^{-\gamma\left(r-r_{0}\right)}-1\right)^{2},
$$

bond bending was controlled by a harmonic cosine potential:

$$
U_{\text {bend }}=\frac{1}{2} K_{\text {bend }}\left(\cos \Theta-\cos \Theta_{0}\right)^{2},
$$

and bond torsions with a twofold torsion potential:

$$
U_{\text {tors }}=\frac{1}{2} K_{\text {tors }}[1-\cos (2 \phi)] \text {. }
$$

The equilibrium bond length $r_{0}$ and the equilibrium bend angle $\Theta_{0}$ were set to the values of the rigid CNT. The stiffness constant $\gamma$ and the force constants $K_{i}$ are $\gamma$ $=2.1867 \AA^{-1}, K_{\mathrm{vib}}=478.9 \mathrm{~kJ} \mathrm{~mol}^{-1}, K_{\text {bend }}=562.2 \mathrm{~kJ} \mathrm{~mol}^{-1}$, and $K_{\text {tors }}=25.12 \mathrm{~kJ} \mathrm{~mol}^{-1}$ respectively. ${ }^{51}$

In this work no intermolecular carbon-carbon interactions were used. Comparisons with results where these interactions were taken into account showed that they have no influence. Due to the CNT geometry they almost cancel out.

\section{Fluid-carbon and fluid-fluid interactions}

Intermolecular interactions were modeled with the shifted and truncated Lennard-Jones (LJ) 12-6 potential, for which a cutoff radius of $14 \AA$ was used. The fluid molecules were modeled as united atoms. The LJ parameters for methane $\left(\sigma_{\mathrm{CH}_{4}}=3.73 \AA, \epsilon_{\mathrm{CH}_{4}} / k_{B}=148 \mathrm{~K}\right)$ were taken from Ref. 52. The LJ parameters for helium $\left(\sigma_{\mathrm{He}}=2.551 \AA, \epsilon_{\mathrm{He}} / k_{B}\right.$ $=10.22 \mathrm{~K})$ and for sulfur hexafluoride $\left(\sigma_{\mathrm{SF}_{6}}=5.128 \AA\right.$, $\left.\epsilon_{\mathrm{SF}_{6}} / k_{B}=222.1 \mathrm{~K}\right)$ were taken from Ref. 53. The carbon LJ 

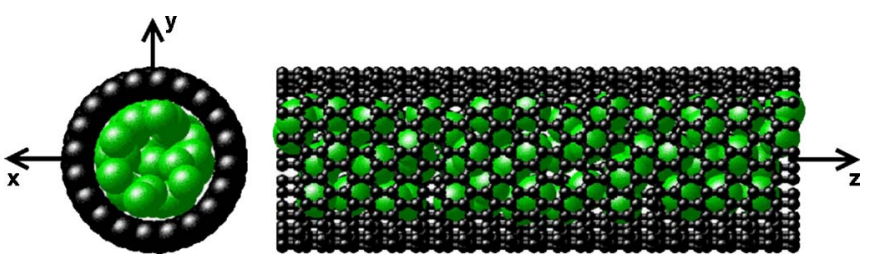

FIG. 1. (Color online) Sketch of the carbon nanotube methane system.

parameters $\left(\sigma_{\mathrm{C}}=3.4 \AA, \epsilon_{\mathrm{C}} / k_{B}=28 \mathrm{~K}\right)$, which are widely accepted for carbon, ${ }^{14}$ were taken from Ref. 54 . The LorentzBerthelot mixing rules were used to determine the interactions of unlike molecular centers.

\section{COMPUTATIONAL DETAILS}

Simulations of a rigid CNT allow the use of interpolation grids for the carbon-fluid interactions. Grid resolutions between 0.028 and $0.049 \AA$ were used (depending on the CNT size). Dubbeldam et al. ${ }^{55}$ and Tepper and Briels ${ }^{56}$ showed that using too few guest particles results in inaccurate diffusion coefficients. Hence, for simulating low loadings one has to increase the size of the CNT. In the case of flexible CNTs this leads to impractical simulation times. To overcome this problem, in all simulations using a flexible pore the length was set to $5.1 \mathrm{~nm}$, but the simulation box length was set to the required value (specified by loading and number of particles). To simulate all fluid particles inside the tube at the correct loading we used different box lengths for the periodic boundary conditions when applied to the carbon-fluid and the fluid-fluid interactions. Each simulation was performed with 64 methane molecules. To confirm that 64 fluid particles were sufficient, some simulations were repeated with twice as many molecules and double the tube length. These simulations produced the same results, so the choice of 64 guest molecules was justified. Zero loading simulations were performed with 64 ideal (noninteracting) fluid particles.

The equations of motion were integrated by the velocity Verlet algorithm ${ }^{57}$ (time step $\Delta t=2 \mathrm{fs}$ ). In addition, for flexible CNT simulations, the reversible reference system propagation algorithm ${ }^{57,58}$ was used, with the short time step
$\left(\Delta t_{S}=0.5 \mathrm{fs}\right)$ for the bond stretch and the long time step $\left(\Delta t_{L}=2 \mathrm{fs}\right)$ for the remainder of the interactions. The particle dynamics were followed for $1-1.5 \mu$ s for cases where the CNT was rigid. In flexible CNT simulations the particle dynamics were followed for $400 \mathrm{~ns}$. The sampling phase was preceded by an equilibration phase, where the particle trajectories were calculated for $0.5-1 \mathrm{~ns}$ depending on the system. To keep the flexible CNT at a constant temperature the NoséHoover chain (NHC) method was used ${ }^{59}$ and the adsorbed fluid was thermalized by the flexible CNT. On the contrary, in rigid CNT simulations the fluid was coupled to a thermostat, either the LA-IFC thermostat or the NHC thermostat. All self-diffusion coefficients $D_{S}$ were calculated for a motion parallel to the pore axis (see Fig. 1) using the Einstein definition: ${ }^{57}$

$$
D_{S}=\frac{1}{2} \lim _{t \rightarrow \infty} \frac{d}{d t}\left\langle[z(t)-z(0)]^{2}\right\rangle .
$$

At short times the velocity of the particles is not affected by the forces, so a nondiffusive behavior is observed (the meansquared displacement is not linear in time). It takes a finite amount of time before the diffusive regime is reached. ${ }^{60}$ Due to the smoothness of a carbon nanotube, this time is much larger than in other nanoporous materials (e.g., zeolites). Two examples (low loading and high loading) are shown in Fig. 2. We applied the linear fit to the mean-squared displacement (MSD) between 1 and 10 ns. The choice of starting at $1 \mathrm{~ns}$ is somewhat arbitrary. It ensures that for all the systems we studied the normal-mode regime was reached. Alternatively, it would be possible to find a starting point more systematically. ${ }^{18}$ To check the accuracy of the results, the diffusion coefficients calculated from the second moment [Eq. (4)] were compared to results calculated from the fourth moment. ${ }^{61}$ For all simulations the agreement was excellent. Errors were calculated by averaging over five independent simulations. In most figures, the error bars are smaller than the symbol size.

To distinguish between the different simulation methods we adopt the following conventions. Simulations including a fully flexible carbon nanotube are denoted as "flexible" CNT

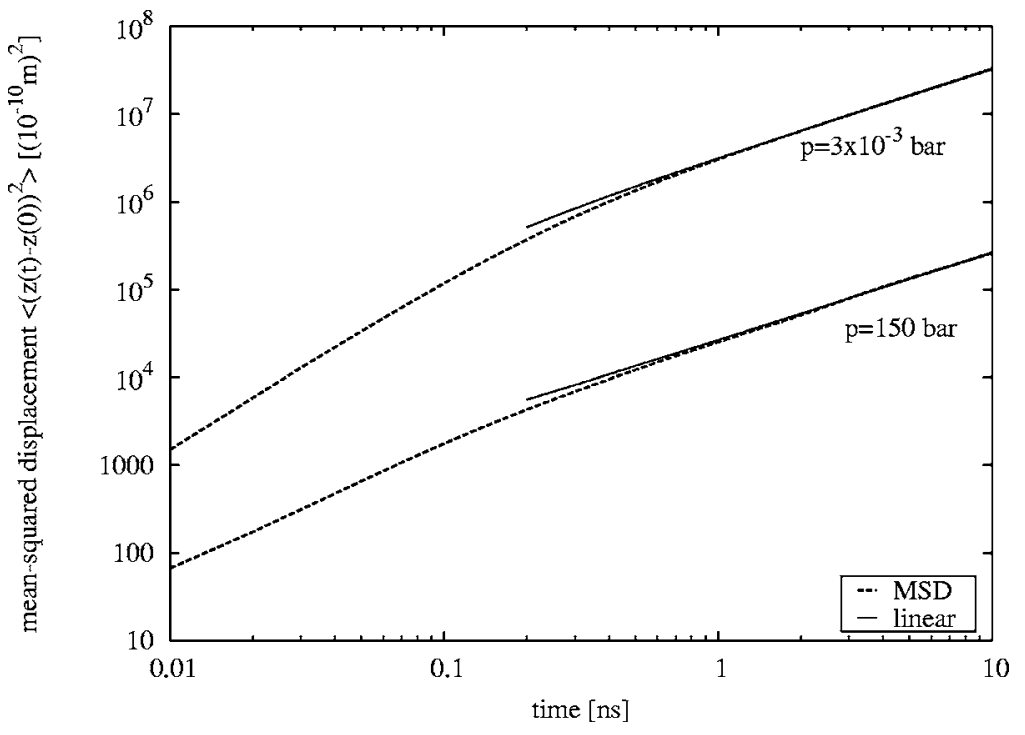

FIG. 2. Mean-squared displacement (MSD) for methane inside a $(20,0) \mathrm{CNT}$ at $300 \mathrm{~K}$. One MSD corresponds to a bulk pressure of $3 \times 10^{-3}$ bars (low loading), and the other to a bulk pressure of 150 bars (high loading). The linear fits were applied between 1 and $10 \mathrm{~ns}$. 


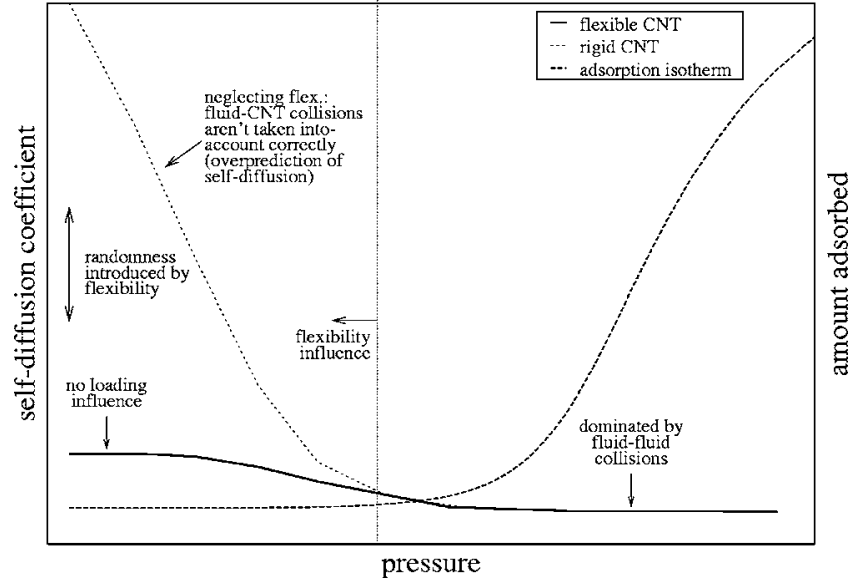

FIG. 3. Schematic sketch of the carbon nanotube flexibility influence on self-diffusion.

simulations; simulations with a rigid CNT that take the flexibility into account via the interface-fluid collision thermostat are termed "LA-IFC" simulations, while rigid CNT simulations where flexibility is neglected (fluid particles coupled to the NHC thermostat) are "rigid CNT" simulations.

\section{THE EFFECT OF FLEXIBILITY ON DIFFUSION}

\section{A. Theoretical aspects}

Whether it is reasonable to assume a rigid lattice in adsorption $^{46}$ or diffusion studies ${ }^{47}$ is far from being understood. An obvious hypothesis is that only in the case of narrow passages is flexibility very important, while in the case of gas molecules in smooth nanoporous materials, e.g., carbon nanotubes, or other nanoporous materials, a rigid lattice is a very reasonable approximation. We have shown that the flexibility of a smooth pore cannot be neglected in the low loading regime. ${ }^{15}$ Simulations in CNTs, or certain other nanoporous materials, that neglect this influence can overpredict the self-diffusion by orders of magnitude (see Fig. 3). Comparisons with the related adsorption isotherms show that the results start to deviate at approximately the transition to the Henry regime. By definition adsorbate-adsorbate interactions are negligible in this regime. ${ }^{62}$

To understand why CNT flexibility has a marked influence on self-diffusion at low loadings, we must consider wall-fluid collisions in detail. At low loadings interactions with other fluid particles are negligible. If the wall is perfectly smooth, the collisions of the gas particles are completely elastic (or specular) and the component of the velocity parallel to the wall remains constant. Hence the parallel motion is ballistic. If, on the other hand, we have a sufficiently corrugated wall, these corrugations introduce sufficient randomness in the collisions to observe a diffusive behavior without the need for collisions with other adsorbed gas particles. CNTs are very smooth and are therefore closer to a perfectly smooth wall. However, in a real nanotube there are always some thermal fluctuations of the wall atoms that interact with the adsorbed molecules. These fluctuations are a source of randomness (increased roughness and diffuse collisions) that will lead to a diffusive behavior. Since at low loadings this effect dominates the introduction of randomness by fluid-fluid collisions, one would observe a constant diffusion coefficient in the limit of low density (see Fig. 3).

Tuzun et al. ${ }^{37}$ observed already in 1996 an influence of flexibility on dynamics inside CNTs. Other recent publications deal with the influence of interface-fluid collisions on diffusion. $^{12-14,16,63,64}$ In a series of publications Sokhan et al. investigated the boundary conditions for fluid flow in carbon nanotubes $^{14,16}$ and slit pores. ${ }^{63,64}$ Among other things they showed that a higher degree of thermalization (diffuse reflection) leads to lower self-diffusion coefficients. ${ }^{16}$ The same trend is observed by Arya et al. ${ }^{13}$ We use this coherence to introduce the flexibility influence in a rigid CNT simulation. A further observation is the minor influence of flexibility at high loadings. ${ }^{16}$

From a computational point of view, simulations approaching the low density limit are surprisingly expensive. To have a certain number of particles present in a molecular dynamics simulation (essential to perform the simulations correctly $^{55,56}$ ), one needs an increasingly long nanotube to reach the low density limit. This presents no difficulty if one assumes a rigid substrate. However, if one has a material in which the flexibility cannot be ignored and a full atom simulation of the material is required, the calculation becomes many orders of magnitude more expensive. It is completely dominated by calculating the dynamics of the substrate. Therefore, most simulation studies use a rigid lattice.

In the next section a novel and very efficient algorithm to avoid the expensive flexible simulations is explained in detail.

\section{B. The Lowe-Andersen thermostat for interface-fluid collisions}

The fact that flexibility has a significant influence on diffusion in CNTs motivated us to introduce an alternative simulation technique that incorporates this influence into a simulation of a rigid CNT. ${ }^{15}$ The important aspect of this algorithm is that it is as efficient as molecular simulations that neglect the flexibility influence while allowing us to take into account the effect of flexibility on the diffusion. The central idea of our algorithm is that due to collisions with the wall, some randomness is introduced into the system. By simulating a CNT as a flexible framework this randomness is treated exactly, whereas in our algorithm it is treated stochastically. Each fluid particle that hits the wall has a probability of exchanging momentum.

In reality the carbon atoms of a CNT undergo thermal fluctuations. During a gas-solid collision these fluctuations introduce some randomness to the motion of the fluid molecules. This effect can be mimicked stochastically, such that during a gas-CNT collision the fluid molecule has a probability of exchanging momentum. This is similar in spirit to the Lowe-Andersen (LA) thermostat for constant temperature (bulk) dissipative particle dynamics simulations. ${ }^{65,66}$ In the original LA thermostat the particles are thermalized during a particle-particle collision. We modified the thermostat in such a way that it works on CNT-fluid collisions such that the influence of a flexible CNT is reproduced. ${ }^{15}$ This modification is not limited to CNT systems. Generally speaking, 
this modified LA thermostat thermalizes the fluid molecules via interface-fluid collisions. To mimic the influence of a flexible CNT with this LA-IFC thermostat the parameters are obtained directly from a simulation of the fully flexible system. The flexible simulations necessary are very short simulations compared to the simulations required to compute the diffusion coefficients.

The LA thermostat works on pairs of particles. A new relative velocity for each pair of particles is taken from a Maxwell distribution, so that the total momentum is conserved. ${ }^{65}$ In real systems, heat is transferred via the system boundaries only, i.e., in our case via the carbon nanotube. On the microscopic level the energy transfer takes place during a collision of a gas molecule with the CNT. This can be mimicked stochastically via a LA thermostat that only acts when there are fluid-wall collisions. In the simulations the carbon atoms are kept fixed (rigid CNT), so only the velocity of the fluid particle is changed. The momentum is not conserved. If the shortest distance between a particle and the wall (defined by the positions of the carbon nuclei) is shorter than $r_{\text {LA }}$, the velocity of a molecule is updated with a probability $\Gamma \Delta t$ and the new velocity is given by

$$
v_{f, \alpha}^{\text {new }}=v_{f, \alpha}^{\text {old }}+\frac{\mu}{m_{f}}\left(\xi_{1} \sqrt{\frac{k_{B} T}{m_{\mathrm{C}}}}-v_{f, \alpha}^{\text {old }}\right)+\xi_{2} \sqrt{\frac{\mu k_{B} T}{m_{f}^{2}}},
$$

where $\alpha$ represents the direction of the velocity $v, m$ is the mass of a particle, the subscript $f$ indicates a fluid molecule, the subscript $\mathrm{C}$ stands for carbon, $\mu=m_{f} m_{\mathrm{C}} /\left(m_{f}+m_{\mathrm{C}}\right)$ is the reduced mass, $k_{B}$ is the Boltzmann constant, $T$ is the temperature, and $\xi_{1}$ and $\xi_{2}$ are independent random numbers from a Gaussian distribution with zero mean and unit variance.

The collision frequency $\Gamma$ and the LA radius $r_{\mathrm{LA}}$ (also termed collision radius) have to be chosen in advance. This is done in a manner such that the LA-IFC thermostat optimally describes a truly flexible CNT. The CNT geometry (see Fig. 1) results in a different mobility of the carbon atoms in different directions. ${ }^{16}$ Orthogonal to the tube axis ( $x$ and $y$ directions), the guest molecules are restricted by the pore walls and, due to the relatively small pore radius, the decorrelation of the velocities in these directions is much faster than that of the velocity parallel to the pore axis ( $z$ direction). Although a collision itself is not direction dependent, we account for the different influences orthogonal and parallel to the pore axis by using different thermostat collision frequencies. The orthogonal components of the particle velocity are updated [see Eq. (5)] with a probability $\Gamma_{x y} \Delta t$, and the parallel component is updated with a probability $\Gamma_{z} \Delta t$. Here $\Delta t$ is the time step size. The update is performed only if the distance between the particle and the CNT is shorter than a distance $r_{\text {LA }}$ (see Fig. 4).

The collision frequencies can be computed from the velocity autocorrelation function (VACF) of the fully flexible system. To relate $\Gamma$ to the VACF we start with the Langevin equation, in the form of ensemble averages:

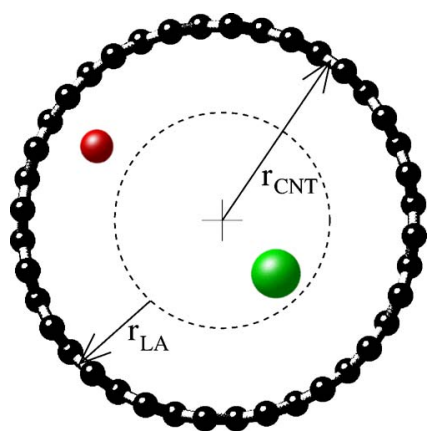

FIG. 4. (Color online) Illustration of the Lowe-Andersen radius (collision radius) $r_{\mathrm{LA}}$. One particle (bigger) is nonthermalized; the velocities of the other particle are recalculated with certain probabilities. The $z$ component is recalculated with the probability $\Gamma_{z} \Delta t$. Whereas, the $x$ and $y$ components are recalculated with the probability $\Gamma_{x y} \Delta t$.

$$
\frac{\partial\langle v(t)\rangle}{\partial t}=\frac{1}{m_{f}}\left\langle f^{\text {friction }}(t)\right\rangle+\frac{1}{m_{f}}\left\langle f^{\text {random }}(t)\right\rangle,
$$

where the total force is divided into a friction force (systematic) and a random force (fluctuating/noise). A reasonable assumption is that the random force arises from white noise (Markovian noise): ${ }^{67}$

$$
\left\langle f^{\text {random }}(t)\right\rangle=0
$$

The average of the frictional force can be rewritten for the zero loading limit:

$$
\left\langle f^{\text {friction }}(t)\right\rangle=\left\langle P_{\mathrm{IFC}} m_{f} \frac{\Delta v}{\Delta t}\right\rangle,
$$

with the probability $P_{\mathrm{IFC}}$ for an interface-fluid collision:

$$
P_{\mathrm{IFC}}=\Gamma \Delta t
$$

By substituting the last three equations into Eq. (6) and multiplying both sides with $v(0)$ we have

$$
\frac{\partial\langle v(t) v(0)\rangle}{\partial t}=\Gamma\langle\Delta v v(0)\rangle .
$$

For fluid-wall collisions the particle velocity is updated using Eq. (5); this equation can be rewritten as

$$
\Delta v=v^{\text {new }}-v^{\text {old }}=\frac{\mu}{m_{f}}\left(v_{w}-v(t)\right)+\Delta_{12},
$$

with

$$
v_{w}=\xi_{1} \sqrt{\frac{k_{B} T}{m_{\mathrm{C}}}}
$$

and

$$
\Delta_{12}=\xi_{2} \sqrt{\frac{\mu k_{B} T}{m_{f}^{2}}} .
$$

Combining Eqs. (10) and (11) leads to 


$$
\begin{aligned}
\frac{\partial\langle v(t) v(0)\rangle}{\partial t}= & \Gamma\left[\frac{\mu}{m_{f}}\left(-\langle v(t) v(0)\rangle+\left\langle v_{w} v(0)\right\rangle\right)\right. \\
& \left.+\left\langle\Delta_{12} v(0)\right\rangle\right] .
\end{aligned}
$$

$v_{w}$ and $\Delta_{12}$ are drawn from independent distributions with zero mean [see Eqs. (12) and (13)], so there is no correlation with $v(0)$ and the two last terms vanish:

$$
\begin{aligned}
& \left\langle v_{w} v(0)\right\rangle=0, \\
& \left\langle\Delta_{12} v(0)\right\rangle=0 .
\end{aligned}
$$

So Eq. (14) is simplified to

$$
\frac{\partial\langle v(t) v(0)\rangle}{\partial t}=-\Gamma \frac{\mu}{m_{f}}\langle v(t) v(0)\rangle .
$$

This is a linear first-order differential equation with the solution

$$
\langle v(0) v(t)\rangle=\left\langle v(0)^{2}\right\rangle \exp \left[-\frac{\Gamma \mu}{m_{f}} t\right] .
$$

The last equation relates the collision frequency $\Gamma$ to the VACF. A central result is that it connects a parameter of our LA-IFC thermostat to an easily computable property in a flexible system. Thus, this equation can be used to obtain the collision frequencies. By comparing Eq. (18) to the result of the Langevin theory,

$$
\langle v(0) v(t)\rangle=\frac{3 k_{B} T}{m_{f}} \exp \left[-\frac{\gamma t}{m_{f}}\right],
$$

the relation between the friction coefficient $\gamma$ and the collision frequency is obtained:

$$
\gamma=\Gamma \mu \text {. }
$$

As discussed above, the CNT geometry leads to two different collision frequencies for CNTs. To calculate the parallel collision frequency $\Gamma_{z}$ Eq. (18) is rewritten for the $z$ direction:

$$
\left\langle v_{z}(0) v_{z}(t)\right\rangle=\left\langle v_{z}(0)^{2}\right\rangle \exp \left[-\frac{\Gamma_{z} \mu}{m_{f}} t\right] .
$$

The VACF, required to calculate $\Gamma_{z}$, is calculated from a simulation of 64 ideal-gas particles inside a CNT of $5.1 \mathrm{~nm}$ length. All parallel collision frequencies are averages over five independent simulations. Of course, we have introduced the LA-IFC thermostat to avoid flexible CNT simulations. However, a fit to the initial VACF is enough, so the flexible simulations to obtain the VACFs are short (particularly compared to simulations necessary to calculate diffusion coefficients). The parallel collision frequency $\Gamma_{z}$ is fitted to the VACF in the range $0-100 \mathrm{ps}(0-1000 \mathrm{ps}$ for some systems), and the particle dynamics are followed for 8-16 ns (depending on the system). A comparison with the results of a much longer simulation justifies these choices. Of course, it is possible to rewrite Eq. (18) for $\Gamma_{x y}$ (or for the individual directions, respectively). Due to the high collision frequency in these directions, the VACF decays rapidly to zero. This makes a fitting difficult. So a different procedure is used to obtain the orthogonal collision frequency $\Gamma_{x y}$.
The thermalizing of a gas inside a CNT by the flexible CNT (wall-fluid collisions) is a local process; the adsorbed gas is not coupled to a thermostat. However, the flexible CNT is coupled to a global thermostat (the NHC thermostat). By using local thermalization it is possible to perform a "heating experiment." In this experiment we introduce a discontinuous change in the temperature of the flexible CNT and monitor the time it takes the gas to reequilibrate at the new temperature. The LA-IFC thermostat is a local thermostat, so it is possible to perform the same heating experiment. The orthogonal collision frequency $\Gamma_{x y}$ is chosen so that the flexible nanotube heating curve is reproduced by the LA-IFC thermostat. At this point it is important to note that several alternative methods are possible to fit this parameter. As we found that this procedure works satisfactorily we did not investigate alternatives.

To reproduce the interface-fluid collisions only, it is important that all simulations necessary for the parameter calculation are performed at the zero loading limit [also required by Eq. (8)]. The heating experiment is performed with 64 noninteracting guest particles. First the gas is equilibrated at $100 \mathrm{~K}$ by following the particle dynamics for $0.5-1 \mathrm{~ns}$ depending on the system. After this equilibration the temperature is changed (defined to be at a time $t=0$ ) to a new temperature $T^{\text {new }}$. For flexible CNT simulations the temperature of the NHC thermostat is changed. As this is a global thermostat, the CNT reaches the new temperature suddenly. We note that the Nosé-Hoover masses should be sufficiently low (Nosé-Hoover frequency is sufficiently high) to assure that the heating of the framework is much faster than the heating of the gas. We use a Nosé-Hoover frequency of 10 . Hence, during the complete heating process the CNT essentially has the properties of a flexible CNT at this new temperature. As the collision frequencies are temperature dependent, the new temperature should be the temperature for which one wishes to calculate the frequencies. For heating experiments with the LA-IFC thermostat the desired temperature is changed [temperature in Eq. (5)]. The orthogonal collision frequency is chosen so that the flexible heating curve is reproduced, defined as minimizing the root mean square deviation (rmsd):

$$
\operatorname{rmsd}=\sqrt{\sum_{t}\left[\frac{T_{\text {rigid }}(t)-T_{\text {flex }}(t)}{T_{\text {flex }}(t)}\right]^{2}} .
$$

Another possibility is to calculate the orthogonal frequency directly from the flexible heating curves. Therefore, we first have to consider the energy exchange during a single collision in the LA-IFC thermostat. Kinetic energy is related to the squared velocity; hence we start with

$$
\left\langle\left(v_{\alpha}^{\text {new }}\right)^{2}\right\rangle=\left\langle\left[v_{\alpha}^{\text {old }}+\frac{\mu}{m_{f}}\left(v_{w}-v_{\alpha}^{\text {old }}\right)+\Delta_{12}\right]^{2}\right\rangle .
$$

The cross correlation functions obtained by multiplying the brackets out vanish, and we are left with 


$$
\left\langle\left(v_{\alpha}^{\text {new }}\right)^{2}\right\rangle=f^{2}\left\langle\left(v_{\alpha}^{\text {old }}\right)^{2}\right\rangle+\frac{\mu^{2}}{m_{f}^{2}}\left\langle v_{w}^{2}\right\rangle+\left\langle\Delta_{12}^{2}\right\rangle,
$$

where $f=1-\mu / m_{f}$. This equation can be rewritten in terms of temperatures,

$$
T^{\text {new }}=f^{2} T^{\text {old }}+\frac{\mu^{2}}{m_{f}} \frac{T_{w}}{m_{\mathrm{C}}}+\frac{\mu}{m_{f}} T_{w},
$$

where the superscripts refer to the temperature before (old) and after (new) the collision and $T_{w}$ denotes the temperature of the wall (desired temperature). Rearrangements lead to the simple relation

$$
T^{\text {new }}-T^{\text {old }}=\left(f^{2}-1\right)\left(T^{\text {old }}-T_{w}\right) .
$$

This equation shows to what extent the kinetic energy of a colliding particle is changed by a thermostat collision. During a heating process energy is transferred to the fluid. In simulations using the new thermostat this energy is transferred via the Lowe-Andersen collisions (see last equation); the energy balance is given by

$$
\begin{aligned}
E(t)-E\left(t_{0}\right)= & \int_{t_{0}}^{t}\left\langle N_{\mathrm{eff}}\right\rangle \Gamma_{z} \frac{1}{2} k_{B}\left(f^{2}-1\right)\left(T_{z}(t)-T_{w}\right) d t \\
& +\int_{t_{0}}^{t}\left\langle N_{\mathrm{eff}}\right\rangle \Gamma_{x y} \frac{1}{2} k_{B}\left(f^{2}-1\right)\left(T_{x}(t)-T_{w}\right) d t \\
& +\int_{t_{0}}^{t}\left\langle N_{\mathrm{eff}}\right\rangle \Gamma_{x y} \frac{1}{2} k_{B}\left(f^{2}-1\right)\left(T_{y}(t)-T_{w}\right) d t,
\end{aligned}
$$

with $T_{\alpha}$ a temperature calculated from one component of the velocity vector only (one-dimensional temperature) and $N_{\text {eff }}$ $\left[=P\left(r_{\mathrm{LA}}\right) N_{\text {total }}\right]$ the number of particles localized in the thermalization region. The last equation can be rearranged to obtain the orthogonal collision frequency $\Gamma_{x y}$ from the heating curves $\left[T_{x}(t), T_{y}(t), T_{z}(t)\right]$ and the energy change of a gas inside a flexible carbon nanotube. By plotting $\Gamma_{x y}$ against time an initial time dependence can be observed due to the fast initial response. After this short time the collision frequency becomes independent of time. After equilibration at the new temperature a drift in $\Gamma_{x y}$ is observed; this is due to statistical fluctuations in the temperatures. This approach leads to results comparable to the results obtained by minimizing the rmsd. One procedure, among others, is to first use Eq. (27) and subsequently adjust the result by minimizing Eq. (22).

The time to reequilibrate at the new temperature depends on the type of the gas inside the CNT. For helium, $\mathrm{CH}_{4}$, and $\mathrm{SF}_{6}$ the heating process takes approximately $100 \mathrm{ps}, 200 \mathrm{ps}$, and $1.5 \mathrm{~ns}$, respectively (see Fig. 5). All heating curves are averages over 100 independent simulations. Because heating is a fast process the calculation time is rather small. The influence of the orthogonal collision frequency on the selfdiffusion parallel to the pore axis is very low; e.g., by recalculating the zero loading result for $\mathrm{CH}_{4}$ with $\Gamma_{x y}=6.4$ $\times 10^{11} \mathrm{~s}^{-1}$ instead of $\Gamma_{x y}=3.5 \times 10^{11} \mathrm{~s}^{-1}$, a diffusion coefficient of $18.27 \pm 0.43 \times 10^{-6} \mathrm{~m}^{2} \mathrm{~s}^{-1}$ instead of $18.92 \pm 0.31$ $\times 10^{-6} \mathrm{~m}^{2} \mathrm{~s}^{-1}$ is obtained.

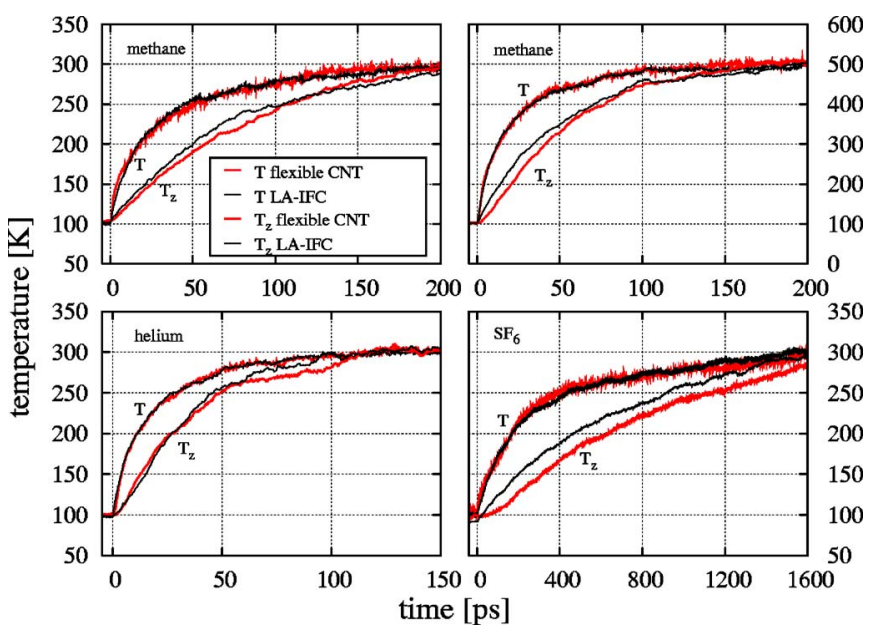

FIG. 5. (Color online) Heating curves for heating ideal gases in a $(20,0)$ carbon nanotube, calculated with a flexible CNT (thick lines) or with the LA-IFC thermostat (thin lines). The LA-IFC curves are calculated with the data sets where the collision radius corresponds to the maximum in the RDF (see Table II). $T$ is the conventional temperature $T=f\left(v_{x}, v_{y}, v_{z}\right)$, and $T_{z}$ the temperature for the $z$ direction only. The temperature switch is defined to take place at a time $t=0 \mathrm{ps}$. All heating curves are averages over 100 independent simulations.

In a molecular simulation it is possible to calculate onedimensional temperatures or one-dimensional heating curves. As these heating curves are different for the orthogonal and parallel directions (during heating), they provide a check for the consistency of the parameters obtained from the fit to the VACF. Figure 5 and Fig. 3 of Ref. 15 show that the LA-IFC thermostat nicely reproduces the heating curves for all directions under various conditions.

Before the LA-IFC heating experiment can be performed $\Gamma_{z}$ has to be calculated and the collision radius has to be chosen in advance. $\Gamma_{z}$ is computed by taking all particles into account [see Eq. (21)], so it corresponds to a LA radius which is equal to the CNT radius $r_{\mathrm{CNT}}$. To recalculate $\Gamma_{z}$ for different $r_{\mathrm{LA}}$ the probability $P\left(r_{\mathrm{LA}}\right)$, which gives the probability that a molecule is located in a spherical shell specified by $r_{\mathrm{LA}}$ (see Fig. 4), is used. Obviously, it is one for a collision radius equal to the CNT radius: $P\left(r_{\mathrm{LA}}=r_{\mathrm{CNT}}\right)=1$. The parallel collision frequency can be recalculated by

$$
\Gamma_{z}\left(r_{\mathrm{LA}}\right)=\frac{\Gamma_{z}\left(r_{\mathrm{LA}}=r_{\mathrm{CNT}}\right)}{P\left(r_{\mathrm{LA}}\right)} .
$$

The probability $P$ can be calculated from the radial distribution function (RDF), which can in turn be calculated in a molecular dynamics or Monte Carlo (MC) simulation. All RDFs needed for this work were computed in MC simulations in the zero loading limit. We stress again that the collision frequencies are collision radius dependent. The fewer the particles involved in the thermalization process (lower $\left.r_{\mathrm{LA}}\right)$, the higher the collision frequencies. A critical discussion on the choice of this parameter set can be found below.

The approach introduced to obtain the LA-IFC parameters leads to a very good representation of $\mathrm{CH}_{4}$ in a flexible CNT, as shown in Ref. 15. In our previous work we have focused on systems that are dominated by lattice vibrations. The more general case is studied in here. Up to now, we have 

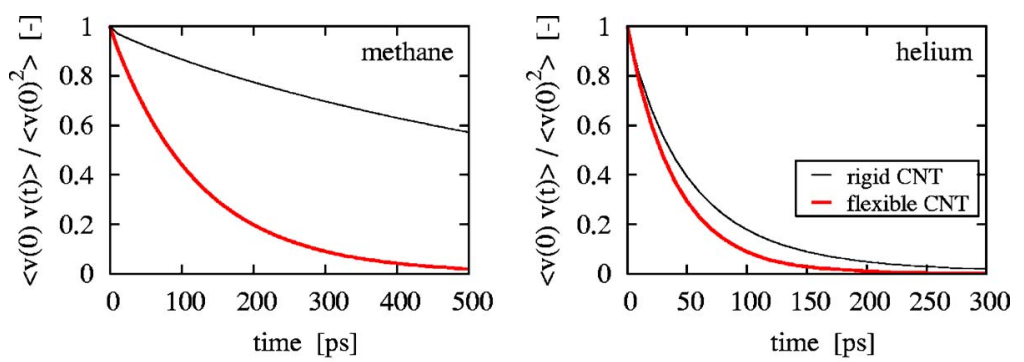

FIG. 6. (Color online) Normalized velocity autocorrelation functions for diffusion inside a $(20,0)$ CNT at $300 \mathrm{~K}$. Comparison of results obtained with a rigid (thin lines) CNT to results obtained with a flexible (thick lines) CNT (top left, methane; top right, helium; bottom left, sulfur hexafluoride; and bottom right, a model fluid with the LJ diameter of helium and all other parameters as those of methane).

assumed that velocity decorrelation arises only from the flexibility of the interface and not from properties present by modeling the interface as rigid. In fact, the velocities are decorrelated by interactions with a rigid interface; otherwise the motion of the particles inside a rigid CNT at the the zero loading limit would be ballistic. The decorrelation effects present in a rigid simulation are also present in a fully flexible interface simulation. So by fitting the collision frequency to the VACF of a flexible CNT and by using this frequency in a rigid CNT simulation, one takes the decorrelations present in a rigid simulation into account twice. For two cases this "double counting" can be neglected. First, the decay of the VACF in the fully flexible CNT is much faster than in the rigid CNT (relative property). Second, the collision frequency is high (absolute property). If the collision frequency is sufficiently high, the thermostat acts so frequently on the fluid particles that it dominates all other decorrelation effects. Both cases are discernible in Fig. 4 of Ref. 15. The difference between the VACFs of methane in a rigid and a flexible CNT is high (see Fig. 6), so the results neglecting the double counting are acceptable. The best reproduction is given by the data set with the highest frequencies. For data sets with lower frequencies the results are slightly underpredicted; the double counting has a small influence. The double counting effect depends on the absolute value of the collision frequency and, more importantly, on the difference of the VACFs (flexible versus rigid). For $\mathrm{SF}_{6}$ the difference of the VACFs (see Fig. 6) is high enough that the effect is never observed. For methane the difference is in a regime where the observability of the effect is determined by the absolute value of the collision frequencies (see Fig. 4 of Ref. 15). The VACF for helium inside a rigid CNT shows a rapid decay, and the additional decorrelation of the velocities caused by flexibility is low. Hence, the double counting cannot be neglected. To answer the question why helium is less influenced by the flexibility compared to methane and $\mathrm{SF}_{6}$, we first simulate methane with the LJ size parameter of helium, second with the LJ well depth of helium, and third with the mass of helium. Only the first of these model fluids shows a similar (small) flexibility influence as does helium (see Fig. 6). For the smaller helium, as well as for the smaller model fluid, the nanotube appears less smooth than for methane. ${ }^{21}$ Therefore the decorrelation is higher. In practice, one can easily test whether lattice vibrations are the dominant contribution. In fact, if such vibrations have a small influence, the rigid lattice simulations already give a good approximation.

To obtain collision frequencies that only take the flexibility effect into account one has to perform one additional step. With the LA-IFC parameters (obtained as described above) one can perform a MD simulation at the zero loading limit. From the VACF of this simulation a collision frequency $\Gamma_{z}^{\text {output }}$ can be calculated [see Eq. (21)]. If the effect of double counting is negligible, this frequency is equal to the frequency $\Gamma_{\text {flex }}$ obtained from the flexible simulation. If not, a new collision frequency can be calculated:

$$
\begin{aligned}
\Gamma_{\mathrm{LA}-\mathrm{IFC}}^{\text {new-input }} & =\left[\Gamma_{\text {flex }}-\left(\Gamma_{\mathrm{LA}-\mathrm{IFC}}^{\text {output }}-\Gamma_{\mathrm{LA}-\mathrm{IFC}}^{\text {input }} P\left(r_{\mathrm{LA}}\right)\right)\right] P\left(r_{\mathrm{LA}}\right)^{-1} \\
& =2 \Gamma_{\mathrm{LA}-\mathrm{IFC}}^{\text {input }}-\Gamma_{\mathrm{LA}-\mathrm{IFC}}^{\text {output }} P\left(r_{\mathrm{LA}}\right)^{-1}
\end{aligned}
$$

where the subscripts flex and LA-IFC indicate in which type of simulation the frequency was obtained/used. To reproduce the flexible CNT results, this recalculation is indispensable for helium (see Fig. 6). Despite the fact that the double counting is irrelevant for methane in most cases and for $\mathrm{SF}_{6}$ in all, recalculation is applied throughout this work. We have recalculated two data sets (highest and lowest collision radii) of our previous diffusion study ${ }^{15}$ with Eq. (29). Figure 7 and Table I show that the reproduction of the flexible results is excellent with the $r_{\mathrm{LA}}=0.78 \mathrm{~nm}$ data set as well. The reproduction with the primal $r_{\mathrm{LA}}=0.36 \mathrm{~nm}$ data set was already satisfactory (see Fig. 4 of Ref. 15), so no improvement is achieved by recalculating this data set. Despite the fact that we can reproduce flexible results with any collision radius, a small $r_{\mathrm{LA}}$ is more reasonable. Remember that the idea was to thermalize at interface-fluid collisions. By using a large collision radius particles that are far away from the wall (no real collision) are thermalized. Hence, if not otherwise stated, we always use a collision radius that corresponds to the maximum of the radial distribution function (at zero loading). An 


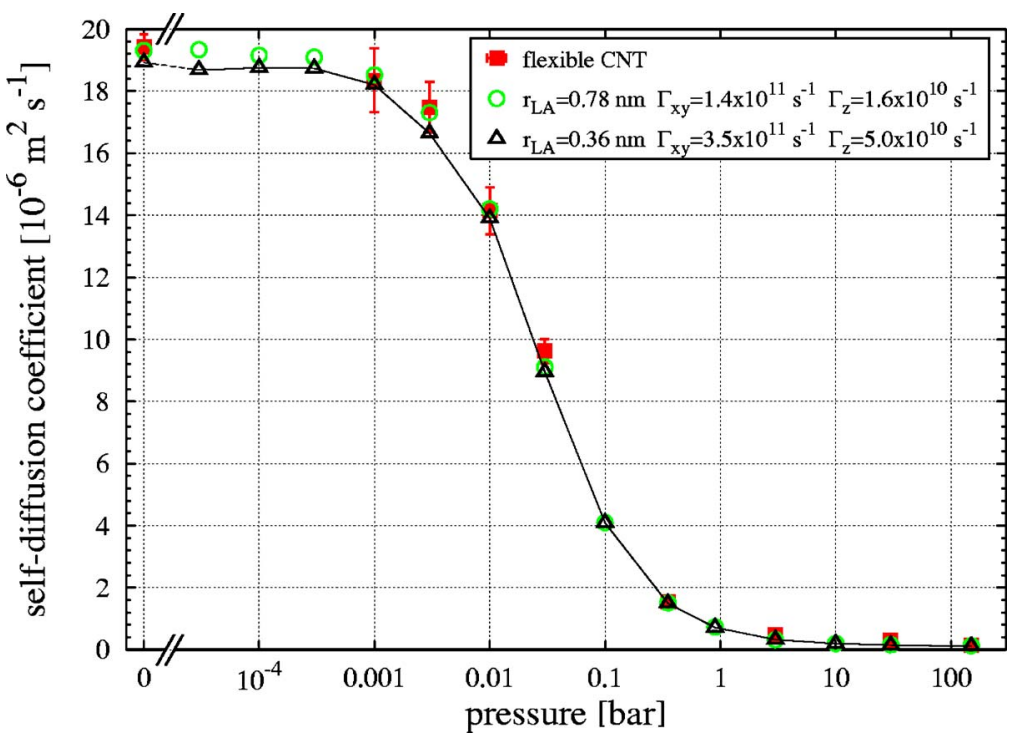

FIG. 7. (Color online) Self-diffusion coefficients of methane inside a $(20,0) \mathrm{CNT}$ at $300 \mathrm{~K}$, obtained with different LA-IFC parameter sets (rigid CNT), compared to results obtained with a flexible CNT. The line is added to guide the eye. outline of all LA-IFC data sets used in this work are given in Table II. Our collision frequencies are of the same order of magnitude as collision frequencies calculated by Sokhan et al. ${ }^{14}$ for nitrogen in a $(16,16) \mathrm{CNT}$.

To summarize, the parametrization proceeds as follows:

(1) Calculate $\Gamma_{z}$ from the VACF of an ideal gas inside a flexible CNT [Eq. (21)].

(2) Choose $r_{\text {LA }}$ and recalculate $\Gamma_{z}$ according to Eq. (28).

(3) Choose $\Gamma_{x y}$ so that the heating curve of an ideal gas thermalized by a flexible CNT is reproduced with these LA-IFC parameters [Eq. (22)].

(4) Use these parameters for a LA-IFC simulation of an ideal gas; calculate $\Gamma_{z}^{\text {output }}$ from the VACF; then recalculate $\Gamma_{z}$ according to Eq. (29); go back to step 3 in order to calculate the related $\Gamma_{x y}$.

(For a discussion of whether or not this step is necessary see the preceding text.)

Implementing the LA-IFC into a molecular simulation is very simple:

- After each time step calculate the distance $r_{\mathrm{IF}}$ of a fluid particle to the interface.

- If $r_{\mathrm{IF}}<r_{\mathrm{LA}}$ (see Fig. 4), update the $z$ component of the velocity with the probability $\Gamma_{z} \Delta t$ and the $x$ and $y$ com-

TABLE I. Self-diffusion coefficients at zero loading inside a $(20,0) \mathrm{CNT}$ at $300 \mathrm{~K}$. Errors are given in the subscripts.

\begin{tabular}{cccc}
\hline \hline Component & Simulation method & $r_{\mathrm{LA}}(\mathrm{nm})$ & $D_{S}\left[10^{-6} \mathrm{~m}^{2} \mathrm{~s}^{-1}\right]$ \\
\hline $\mathrm{CH}_{4}$ & Flexible & $\ldots$ & $19.42_{0.42}$ \\
$\mathrm{CH}_{4}$ & LA-IFC & 0.36 & $18.92_{0.31}$ \\
$\mathrm{CH}_{4}$ & LA-IFC & 0.78 & $19.31_{0.24}$ \\
$\mathrm{CH}_{4}$ & Rigid & $\ldots$ & $159.09_{3.00}$ \\
& & & \\
$\mathrm{He}$ & Flexible & $\ldots$ & $26.18_{1.31}$ \\
$\mathrm{He}$ & LA-IFC & 0.30 & $24.17_{0.57}$ \\
$\mathrm{He}$ & LA-IFC & 0.78 & $25.57_{0.34}$ \\
$\mathrm{He}$ & Rigid & $\ldots$ & $37.91_{1.19}$ \\
\hline \hline
\end{tabular}

ponents with a probability $\Gamma_{x y} \Delta t$; the velocities are updated with Eq. (5).

\section{FLUID-SOLID THERMAL DIFFUSE SCATTERING ALGORITHM}

To avoid flexible host matrix simulations and to ensure that transport occurs under a fluid-solid thermal equilibrium, MacElroy and Boyle ${ }^{50}$ introduced the so-called fluid-solid thermal diffuse scattering (TDS) algorithm. The basic idea is similar to our LA-IFC thermostat; fluid particles are thermalized by fluid-wall collisions. They define a scattering plane at a specific position, and whenever a particle is closer to a wall atom, as specified by this position, the particle is "reflected from the collision plane according to the cosine law of diffuse scattering." 50 The position of the scattering plane is comparable to the collision radius in the LA-IFC algorithm. The main differences are that in the TDS scheme collisions with individual wall atoms are encountered. Hence, multiple collisions (multiple thermalization) for the same particle can occur in one time step. Furthermore, every collision results in a thermalization (diffuse collision), resulting in a collision probability within the scattering plane of unity. The position of the scattering plane is chosen to be at the position of the fluid-wall atom potential minimum.

TABLE II. Parameter sets for the LA-IFC thermostat.

\begin{tabular}{ccccccc}
\hline \hline Component & $\begin{array}{c}T \\
(\mathrm{~K})\end{array}$ & $\mathrm{CNT}$ & $\begin{array}{c}r_{\mathrm{CNT}} \\
(\mathrm{nm})\end{array}$ & $\begin{array}{c}r_{\mathrm{LA}} \\
(\mathrm{nm})\end{array}$ & $\begin{array}{c}\Gamma_{x y} \\
\left(10^{11} \mathrm{~s}^{-1}\right)\end{array}$ & $\begin{array}{c}\Gamma_{z} \\
\left(10^{10} \mathrm{~s}^{-1}\right)\end{array}$ \\
\hline $\mathrm{CH}_{4}$ & 300 & $(15,0)$ & 0.59 & 0.37 & 3.8 & 4.0 \\
$\mathrm{CH}_{4}$ & 300 & $(20,0)$ & 0.78 & 0.36 & 3.5 & 5.0 \\
$\mathrm{CH}_{4}$ & 300 & $(20,0)$ & 0.78 & 0.78 & 1.4 & 1.6 \\
$\mathrm{CH}_{4}$ & 300 & $(25,0)$ & 0.98 & 0.36 & 4.1 & 5.7 \\
$\mathrm{CH}_{4}$ & 500 & $(20,0)$ & 0.78 & 0.36 & 5.0 & 7.5 \\
$\mathrm{CH}_{4}$ & 700 & $(20,0)$ & 0.78 & 0.36 & 6.1 & 10.4 \\
$\mathrm{He}$ & 300 & $(20,0)$ & 0.78 & 0.30 & 6.5 & 3.8 \\
$\mathrm{He}$ & 300 & $(20,0)$ & 0.78 & 0.78 & 1.3 & 0.6 \\
\hline \hline
\end{tabular}




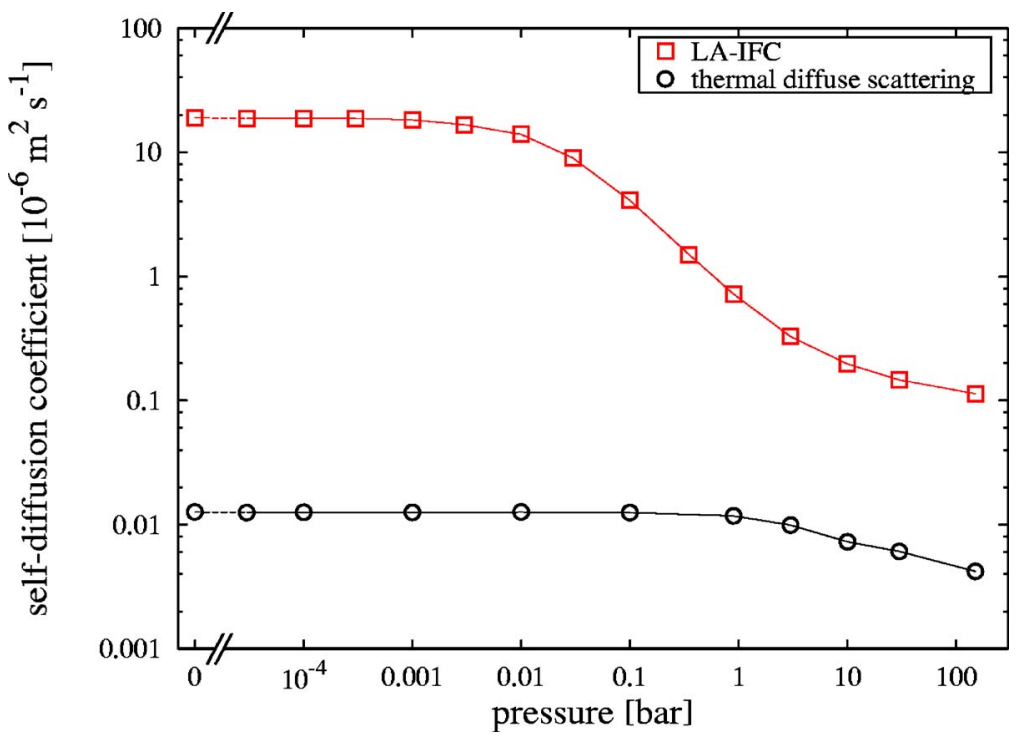

FIG. 8. (Color online) Self-diffusion coefficients of methane inside a $(20,0) \mathrm{CNT}$ at $300 \mathrm{~K}$, obtained with the fluid-solid thermal diffuse scattering algorithm, compared to results obtained with the LA-IFC thermostat. The lines are added to guide the eye.

To compare the results of this fluid-solid thermal diffuse scattering algorithm to those of the Lowe-Andersen interface-fluid collision thermostat, simulations of methane inside a $(20,0) \mathrm{CNT}$ at $300 \mathrm{~K}$ were performed. The TDS algorithm is used as described by MacElroy and Boyle. ${ }^{50}$ Self-diffusion coefficients obtained with the TDS algorithm are three orders of magnitude lower for low loadings and one order of magnitude for high loadings (see Fig. 8). The TDS algorithm thermalizes every particle inside the scattering plane; no thermalization probability is used. This leads to a too rapid thermalization and therefore to an underprediction of self-diffusion. By using a collision radius equal to the position of the methane-carbon potential minimum, we obtain a thermalization probability $\left(\Gamma_{i} \Delta t\right)$ of $4.6 \times 10^{-5}$ for the parallel direction and $3.6 \times 10^{-4}$ for the orthogonal direction. Compared to a probability of 1 in the TDS algorithm, it is obvious that too many thermalizing events occur in the TDS algorithm. The self-diffusion coefficients can be considered as the lower limit for this scattering plane position. Also, the loading dependence (fluid-fluid collision influence) is observed only above 1 bar. On the contrary, treating the flexibility influence correctly the loading dependence is observed approximately from 0.01 bar onwards. Deviations at the high loading regime shows that the wall-fluid collisions have an influence over the entire loading range; this is usually not the case (e.g., see Figs. 9 of this work and 2 of Ref. 15). We also note that heating curves obtained with the TDS algorithm display a much too rapid heating (data not shown).

The only parameter in the TDS algorithm is the location of the scattering plane. Shifting this location closer to the wall results in less thermalization. But for this a method has to be developed to choose this position systematically, such that correct self-diffusion coefficients are obtained. Furthermore, the TDS algorithm does not distinguish collisions parallel to the pore axis and orthogonal to the pore axis.

We note that we have investigated the influence of the TDS algorithm only on self-diffusion. Most works in which the TDS algorithm is used deal with transport diffusion. ${ }^{50,68}$ It is observed that self- and transport diffusivities in CNTs are different by orders of magnitude. ${ }^{10,23}$ So it could be that the TDS algorithm has less influence on transport diffusion than on self-diffusion. For instance, it was shown that velocity scaling has no influence on gas effusion from slit pores, compared to a flexible pore model. ${ }^{69}$

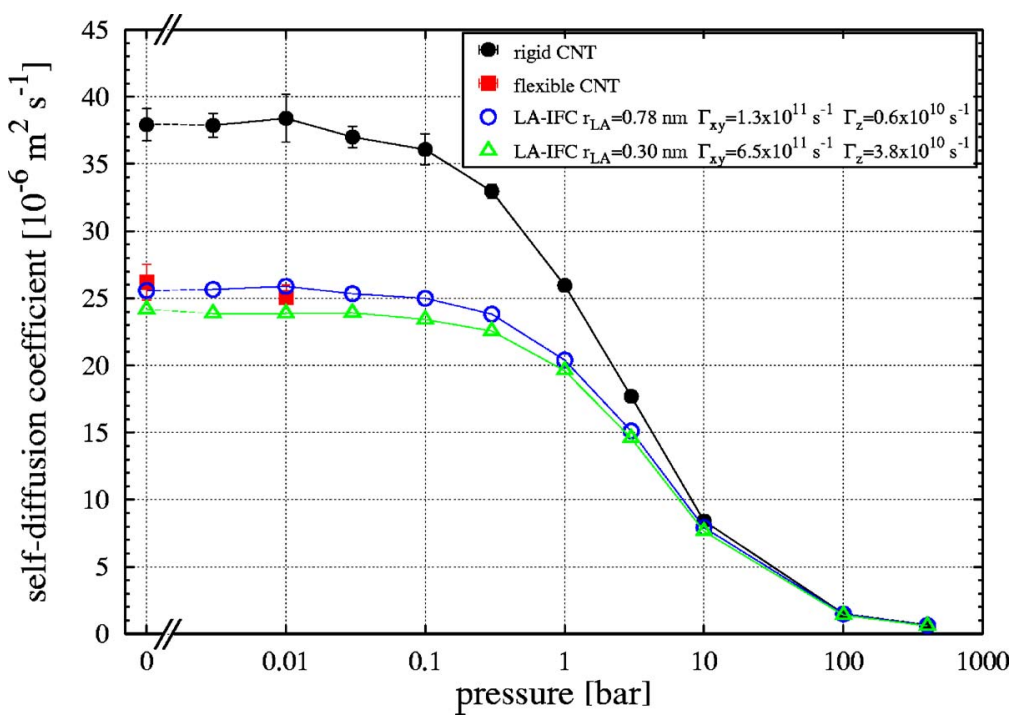

FIG. 9. (Color online) Self-diffusion coefficients of helium inside a $(20,0) \mathrm{CNT}$ at $300 \mathrm{~K}$, calculated with a flexible CNT, with a rigid nanotube, and with different LA-IFC parameter sets. The lines are added to guide the eye. 


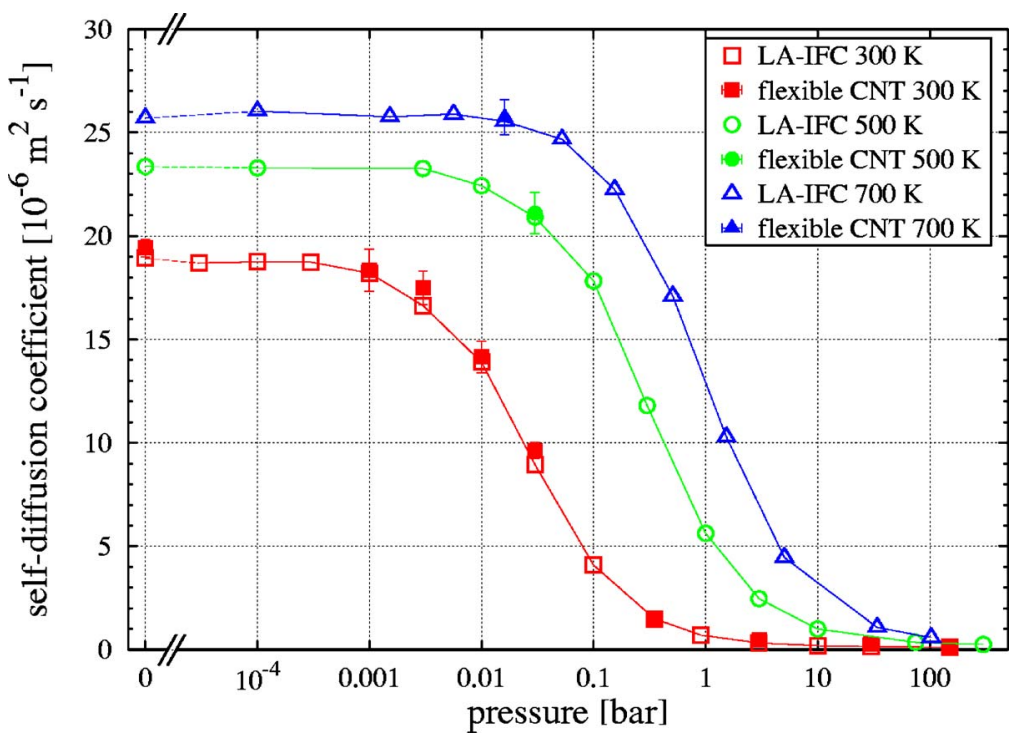

FIG. 10. (Color online) Self-diffusion coefficients of methane inside a $(20,0) \mathrm{CNT}$ at various temperatures, calculated with the LA-IFC thermostat, compared to results obtained with a flexible CNT. The lines are added to guide the eye.

\section{SELF-DIFFUSION IN SINGLE WALLED CARBON NANOTUBES}

In this section we show results for self-diffusion of methane and helium in various carbon nanotubes at different conditions. As mentioned above, the self-diffusion of helium in carbon nanotubes is less influenced by carbon nanotube flexibility compared to that of methane. A plateau region in the limit of low loadings is already observed by simulating the CNT as a rigid framework (Fig. 9). This physically reasonable plateau region is not obtained if methane is adsorbed. ${ }^{15}$ Even though the deviation is not orders of magnitude, the flexibility cannot be neglected. In the zero loading limit the deviation is still 40\% (see Table I). As observed for methane, ${ }^{15}$ the flexibility has no influence in the high loading region. Here the diffusion process is completely determined by fluid-fluid collisions. The loading dependence of the selfdiffusion of helium inside a $(20,0) \mathrm{CNT}$ at $300 \mathrm{~K}$ is again simulated with two different LA-IFC parameter sets. In the first set we use a collision radius which is equal to the CNT radius, and in the second one we use $r_{\mathrm{LA}}$ according to the maximum of the RDF. Both data sets give an excellent re- production of the flexible results (see Fig. 9 and Table I). The helium self-diffusion coefficients for low loadings are higher than for the corresponding methane system. This is because of a smaller mass and a smaller size compared to those of methane.

To investigate the temperature influence on selfdiffusion in carbon nanotubes, we have simulated methane inside a $(20,0) \mathrm{CNT}$ at three different temperatures (see Fig. 10). The reproduction of the simulations of flexible CNTs by the LA-IFC simulations is excellent for all three temperatures. As expected, the higher the temperature, the higher the self-diffusion coefficients. This difference is more pronounced at low loadings. At high loadings, where the mobility of the fluid molecules is lower, the difference almost vanishes.

In Fig. 11 the loading dependence of self-diffusion is shown for three different pore sizes (see Table II). For loadings near saturation the self-diffusion coefficients are almost the same for all pores. Here the motion is dominated by fluid-fluid collisions, and wall-fluid collisions (and therefore the pore structure) have only a small influence. In the zero

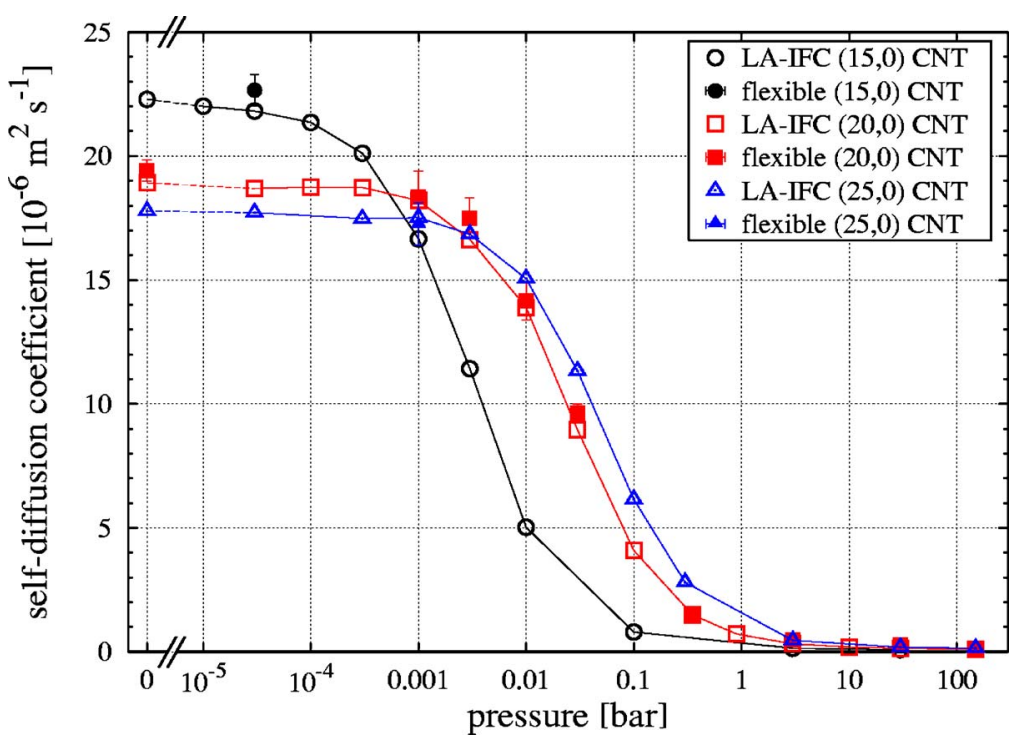

FIG. 11. (Color online) Self-diffusion coefficients of methane inside various CNTs at $300 \mathrm{~K}$, calculated with the LA-IFC thermostat, compared to results obtained with a flexible CNT. The lines are added to guide the eye. 
loading limit the fastest diffusion is observed in the smallest CNT. The $(15,0)$ CNT is comparable in size with a $(8,8)$ CNT (armchair structure, $r_{\mathrm{CNT}}=0.54 \mathrm{~nm}$ ). Bhide and Yashonath $^{34}$ studied the mobility of methane in this CNT (using the rigid CNT assumption). By fitting the MSD to the relation $\left\langle[z(t)-z\langle 0)]^{2}\right\rangle=C t^{\alpha}$ for the first $50 \mathrm{ps}$ only, they found $\alpha=1.811$. They performed the simulations at a loading of 0.40 molecules $/ \mathrm{nm}$, and they expect the motion to go over to the normal-mode diffusion for higher loadings. Analyzing the MSD for methane inside a $(15,0)$ CNT at 0.1 bar (corresponding to a loading of 0.21 molecules $/ \mathrm{nm}$ ) for $0-50 \mathrm{ps}$, we obtain $\alpha=1.32$ for LA-IFC simulations and $\alpha=1.34$ for rigid simulations. By analyzing the MSD between 1 and $10 \mathrm{~ns}$ (as done throughout this work) we obtain $\alpha=0.99$ for LA-IFC simulations and $\alpha=0.99$ for rigid simulations. Clearly, the observation of "superdiffusive motion" in our case can be attributed to limiting the analysis of the MSD to the first few picoseconds. Bhide and Yashonath $^{34}$ used a slightly smaller CNT and a different force field. It is therefore necessary to repeat these simulations for longer times to investigate whether also in this system normal diffusion is recovered. Most probably this applies also to their other studies. ${ }^{33,35,36}$ It is well known that at short times the MSD always displays a ballistic regime. ${ }^{60}$ Furthermore, the time it takes to go over to the normal-mode diffusion is, for particles confined in CNTs, much higher than for particles confined in zeolites (due to the smoothness of CNTs). Hence, it is reasonable to find superdiffusive motion when analyzing the MSD for only the first $50 \mathrm{ps}$.

\section{SUMMARY}

In this paper we have discussed our recently introduced novel algorithm ${ }^{15}$ to stochastically introduce the effects of a flexible CNT into a simulation using a rigid CNT. This LAIFC thermostat avoids simulations employing a flexible CNT. The simulations are significantly faster. Depending on the loading and details of the implementation, factors 25 to 5 orders of magnitude are estimated. Simulations with this new algorithm lead to the same self-diffusion coefficients and give the same heat transfer across the rigid interface as across a flexible interface. To reproduce the flexible CNT results, the parameters of the LA-IFC thermostat are obtained from short flexible CNT simulations. The parameter calculation and the implementation of the algorithm are described in detail in this paper.

Furthermore, the influence of flexibility on self-diffusion in single walled carbon nanotubes was investigated. We showed that the flexibility of a smooth pore cannot be neglected in the low loading regime. A comparison with the adsorption isotherm shows that the results start to deviate approximately at the transition to the Henry regime. By definition, adsorbate-adsorbate interactions are negligible in the Henry regime. ${ }^{62}$ Simulations, in CNTs or certain other nanoporous materials, that neglect this influence ${ }^{10,17-19}$ can lead to an overprediction of the self-diffusion coefficient by orders of magnitude. The computed self-diffusion coefficients in a flexible CNT are still higher than for zeolites, but not at two orders of magnitude as suggested by a simulation in a rigid
CNT. They are also lower than the corresponding diffusion coefficients in the gas phase. The flexibility influence is reduced for an increased relative roughness of the rigid pore wall.

The small vibrations of the carbon atoms have a significant influence on the self-diffusion at low loadings in CNTs. This requires very expensive simulations. We have shown that these simulations can be avoided by using the LA-IFC thermostat. Interestingly, the situation is different for zeolites where sophisticated techniques are necessary to simulate slow diffusion at high loadings. ${ }^{70}$ These two cases can be counted as the extreme cases of diffusion in confinement: diffusion at low loadings in CNT as a fast process and diffusion at high loadings in zeolites as a slow process.

In addition, the influence of temperature and pore size was investigated. The loading dependence of self-diffusion in carbon nanotubes for methane in various CNTs at three different temperatures was computed. The loading dependence of self-diffusion for helium in a $(20,0) \mathrm{CNT}$ at $300 \mathrm{~K}$ was also investigated. Due to an increased relative roughness (smaller molecule) of the carbon nanotube surface, the flexibility influence is less than in the case of methane. Nonetheless, there is still an effect, which our methodology reproduces, so long as the procedure we detail for avoiding a double counting is used.

\section{ACKNOWLEDGMENTS}

This work was supported by the Deutsche Forschungsgemeinschaft (DFG) in priority program SPP 1155 . We thank Tina Düren for helpful discussions on the TDS algorithm, and we are grateful to H. Chen, J. K. Johnson, and D. S. Sholl for sending us their manuscript prior to publication.

${ }^{1}$ M. S. Dresselhaus, G. Dresselhaus, and P. Avouris, Carbon Nanotubes Synthesis, Structure, Properties and Applications, Topics in Applied Physics Series Vol. 80 (Springer, Berlin, 2001).

${ }^{2}$ P. J. F. Harris, Carbon Nanotubes and Related Structures New Materials for the Twenty-first Century (Cambridge University Press, Cambridge, 2001).

${ }^{3}$ S. Iijima, Nature (London) 354, 56 (1991).

${ }^{4}$ K. Hata, D. N. Futaba, K. Mizuno, T. Namai, M. Yumura, and S. Iijima, Science 306, 1362 (2004).

${ }^{5}$ W. A. de Heer, P. Poncharal, C. Berger, J. Gezo, Z. Song, J. Bettini, and D. Ugarte, Science 307, 907 (2005).

${ }^{6}$ B. Wei, R. Vajtai, Y. Jung, J. Ward, R. Zhang, G. Ramanath, and P. M. Ajayan, Nature (London) 416, 495 (2002).

${ }^{7}$ B. J. Hinds, N. Chopra, T. Rantell, R. Andrews, V. Gavalas, and L. G. Bachas, Science 303, 62 (2004).

${ }^{8}$ M. J. Casavant, D. A. Walters, J. J. Schmidt, and R. E. Smalley, J. Appl. Phys. 93, 2153 (2003).

${ }^{9}$ A. Srivastava, O. N. Srivastava, S. Talapatra, R. Vajtai, and P. Ajayan, Nat. Mater. 3, 610 (2004).

${ }^{10}$ A. I. Skoulidas, D. M. Ackerman, J. K. Johnson, and D. S. Sholl, Phys. Rev. Lett. 89, 185901 (2002).

${ }^{11}$ C. Wei and D. Srivastava, Phys. Rev. Lett. 91, 235901 (2003).

${ }^{12}$ O. G. Jepps, S. K. Bhatia, and D. J. Searles, Phys. Rev. Lett. 91, 126102 (2003).

${ }^{13}$ G. Arya, H. C. Chang, and E. J. Maginn, Phys. Rev. Lett. 91, 026102 (2003).

${ }^{14}$ V. P. Sokhan, D. Nicholson, and N. Quirke, J. Chem. Phys. 120, 3855 (2004).

${ }^{15}$ S. Jakobtorweihen, M. G. Verbeek, C. P. Lowe, F. J. Keil, and B. Smit, Phys. Rev. Lett. 95, 044501 (2005).

${ }^{16}$ V. P. Sokhan, D. Nicholson, and N. Quirke, J. Chem. Phys. 117, 8531 (2002). 
${ }^{17}$ D. Cao and J. Wu, Langmuir 20, 3759 (2004).

${ }^{18}$ G. Arora, N. J. Wagner, and S. I. Sandler, Langmuir 20, 6268 (2004).

${ }^{19}$ D. M. Ackerman, A. I. Skoulidas, D. S. Sholl, and J. K. Johnson, Mol. Simul. 29, 677 (2003).

${ }^{20}$ H. Chen and D. S. Sholl, J. Am. Chem. Soc. 126, 7778 (2004).

${ }^{21}$ S. K. Bhatia, H. Chen, and D. S. Sholl, Mol. Simul. 31, 643 (2005).

${ }^{22}$ H. Chen and D. S. Sholl, J. Membr. Sci. 269, 152 (2006).

${ }^{23}$ A. I. Skoulidas, D. S. Sholl, and J. K. Johnson, J. Chem. Phys. 124, 054708 (2006).

${ }^{24}$ F. Zhang, J. Chem. Phys. 111, 9082 (1999).

${ }^{25}$ G. Garberoglio and R. Vallauri, J. Mol. Liq. 117, 43 (2005).

${ }^{26}$ Y. Liu and Q. Wang, Phys. Rev. B 72, 085420 (2005).

${ }^{27}$ J. Zheng, E. M. Lennon, H.-K. Tsao, Y.-J. Sheng, and S. Jiang, J. Chem. Phys. 122, 214702 (2005).

${ }^{28}$ S. Supple and N. Quirke, Phys. Rev. Lett. 90, 214501 (2003).

${ }^{29}$ K.-H. Lee and S. B. Sinnott, Nano Lett. 5, 793 (2005).

${ }^{30}$ K.-H. Lee and S. B. Sinnott, J. Phys. Chem. B 108, 9861 (2004).

${ }^{31}$ Z. Mao and S. B. Sinnott, J. Phys. Chem. B 105, 6916 (2001).

${ }^{32}$ Z. Mao and S. B. Sinnott, J. Phys. Chem. B 104, 4618 (2000).

${ }^{33}$ S. Y. Bhide and S. Yashonath, J. Am. Chem. Soc. 125, 7425 (2003).

${ }^{34}$ S. Y. Bhide and S. Yashonath, J. Chem. Phys. 116, 2175 (2002).

${ }^{35}$ S. Y. Bhide and S. Yashonath, J. Phys. Chem. A 106, 7130 (2002).

${ }^{36}$ S. Y. Bhide and S. Yashonath, J. Phys. Chem. B 104, 11977 (2000).

${ }^{37}$ R. E. Tuzun, D. W. Noid, B. G. Sumpter, and R. C. Merkle, Nanotechnology 7, 241 (1996).

${ }^{38}$ J. Jiang, S. I. Sandler, M. Schenk, and B. Smit, Phys. Rev. B 72, 045447 (2005).

${ }^{39}$ J. Jiang, S. I. Sandler, and B. Smit, Nano Lett. 4, 241 (2004).

${ }^{40}$ G. Arora and S. I. Sandler, J. Chem. Phys. 123, 044705 (2005).

${ }^{41}$ K. G. Ayappa, Langmuir 14, 880 (1998).

${ }^{42}$ R. F. Cracknell, Mol. Phys. 100, 2079 (2002).

${ }^{43}$ X. R. Zhang and W. C. Wang, Phys. Chem. Chem. Phys. 4, 3048 (2002).

${ }^{44}$ F. Darkrim and D. Levesque, J. Chem. Phys. 109, 4981 (1998).

${ }^{45}$ K. G. Ayappa, Chem. Phys. Lett. 282, 59 (1998).

${ }^{46}$ T. J. H. Vlugt and M. Schenk, J. Phys. Chem. B 106, 12757 (2002).

${ }^{47}$ P. Demontis and G. B. Suffritti, Chem. Rev. (Washington, D.C.) 97, 2845 (1997).

${ }^{48}$ H. Chen, J. K. Johnson, and D. S. Sholl, J. Phys. Chem. B 110, 1971 (2006).
${ }^{49}$ S. Andreev, D. Reichman, and G. Hummer, J. Chem. Phys. 123, 194502 (2005).

${ }^{50}$ J. M. D. MacElroy and M. J. Boyle, Chem. Eng. J. 74, 85 (1999).

${ }^{51}$ J. H. Walther, R. Jaffe, T. Halicioglu, and P. Koumoutsakos, J. Phys. Chem. B 105, 9980 (2001).

${ }^{52}$ M. G. Martin and J. I. Siepmann, J. Phys. Chem. B 102, 2569 (1998).

${ }^{53}$ B. E. Poling, J. M. Prausnitz, and J. P. O'Connell, The Properties of Gases and Liquids, 5th ed. (McGraw-Hill, New York, 2000).

${ }^{54}$ W. A. Steele, The Interaction of Gaes with Solid Surfaces (Pergamon, Oxford, 1974).

${ }^{55}$ D. Dubbeldam, E. Beerdsen, T. J. H. Vlugt, and B. Smit, J. Chem. Phys. 122, 224712 (2005).

${ }^{56}$ H. L. Tepper and W. J. Briels, J. Chem. Phys. 116, 9464 (2002).

${ }^{57}$ D. Frenkel and B. Smit, Understanding Molecular Simulations: From Algorithms to Applications (Academic, San Diego, 2002).

${ }^{58}$ M. E. Tuckerman, B. J. Berne, and G. J. Martyna, J. Chem. Phys. 97, 1990 (1992)

${ }^{59}$ G. J. Martyna, M. E. Tuckerman, D. J. Tobias, and M. L. Klein, Mol. Phys. 87, 1117 (1996).

${ }^{60}$ D. Chandler, Introduction to Modern Statistical Mechanics (Oxford University Press, New York, 1987).

${ }^{61}$ S. Fritzsche, R. Haberlandt, J. Kaerger, H. Pfeifer, and K. Heinzinger, Chem. Phys. Lett. 198, 283 (1992).

${ }^{62}$ F. Rouquerol, J. Rouquerol, and K. Sing, Adsorption by Powders \& Porous Solids (Academic, San Diego, 1999).

${ }^{63}$ V. P. Sokhan and N. Quirke, Mol. Simul. 30, 217 (2004).

${ }^{64}$ V. P. Sokhan, D. Nicholson, and N. Quirke, J. Chem. Phys. 115, 3878 (2001).

${ }^{65}$ C. P. Lowe, Europhys. Lett. 47, 145 (1999).

${ }^{66}$ C. P. Lowe and M. W. Dreischor, in Novel Methods in Soft Matter Simulations, Lecture Notes in Physics Vol. 640, edited by M. Karttunen, I. Vattulainen, and A. Lukkarinen (Springer, Berlin, 2004), pp. 39-68.

${ }^{67}$ R. Zwanzig, Nonequilibrium Statistical Mechanics (Oxford University Press, Oxford, 2001).

${ }^{68}$ T. Düren, F. J. Keil, and N. A. Seaton, Chem. Eng. Sci. 57, 1343 (2002).

${ }^{69}$ S. Furukawa, A. Fukui, Y. Zhang, and T. Nitta, Mol. Simul. 30, 379 (2004).

${ }^{70}$ E. Beerdsen, B. Smit, and D. Dubbeldam, Phys. Rev. Lett. 93, 248301 (2004). 